\title{
A character on the quasi-symmetric functions coming from multiple zeta values
}

\author{
Michael E. Hoffman \\ Dept. of Mathematics \\ U. S. Naval Academy, Annapolis, MD 21402 USA \\ meh@usna.edu
}

Submitted: May 6, 2008; Accepted: Jul 23, 2008; Published: Jul 28, 2008

Keywords: multiple zeta values, symmetric functions, quasi-symmetric functions, Hopf algebra character, gamma function, $\Gamma$-genus, $\hat{\Gamma}$-genus

Mathematics Subject Classifications: Primary 05E05; Secondary 11M41, 14J32, 16W30, 57R20

\begin{abstract}
We define a homomorphism $\zeta$ from the algebra of quasi-symmetric functions to the reals which involves the Euler constant and multiple zeta values. Besides advancing the study of multiple zeta values, the homomorphism $\zeta$ appears in connection with two Hirzebruch genera of almost complex manifolds: the $\Gamma$-genus (related to mirror symmetry) and the $\hat{\Gamma}$-genus (related to an $S^{1}$-equivariant Euler class). We decompose $\zeta$ into its even and odd factors in the sense of Aguiar, Bergeron, and Sottille, and demonstrate the usefulness of this decomposition in computing $\zeta$ on the subalgebra of symmetric functions (which suffices for computations of the $\Gamma$ and $\hat{\Gamma}$-genera).
\end{abstract}

\section{Introduction}

Let $x_{1}, x_{2}, \ldots$ be a countably infinite sequence of indeterminates, each having degree 1 , and let $\mathfrak{P} \subset \mathbf{R}\left[\left[x_{1}, x_{2}, \ldots\right]\right]$ be the set of formal power series in the $x_{i}$ having bounded degree. Then $\mathfrak{P}$ is a graded algebra over the reals. An element $f \in \mathfrak{P}$ is called a symmetric function if

$$
\text { coefficient of } x_{n_{1}}^{i_{1}} x_{n_{2}}^{i_{2}} \cdots x_{n_{k}}^{i_{k}} \text { in } f=\text { coefficient of } x_{1}^{i_{1}} x_{2}^{i_{2}} \cdots x_{k}^{i_{k}} \text { in } f
$$

for any $k$-tuple $\left(n_{1}, \ldots, n_{k}\right)$ of distinct positive integers, and $f$ is called a quasi-symmetric function if equation (1) holds whenever

$$
n_{1}<n_{2}<\cdots<n_{k} .
$$

The vector spaces Sym and QSym of symmetric and quasi-symmetric functions respectively are both subalgebras of $\mathfrak{P}$, with Sym $\subset$ QSym. Of course Sym is a familiar object, 
for which the first chapter of Macdonald [20] is a convenient reference. The algebra QSym was introduced by Gessel [9], and in recent years has become increasingly important in combinatorics; see, e.g., [24].

A vector space basis for QSym is given by the monomial quasi-symmetric functions, which are indexed by compositions (ordered partitions). The monomial quasi-symmetric function $M_{I}$ corresponding to the composition $I=\left(i_{1}, i_{2}, \ldots, i_{k}\right)$ is

$$
M_{I}=\sum_{n_{1}<n_{2}<\cdots<n_{k}} x_{n_{1}}^{i_{1}} x_{n_{2}}^{i_{2}} \cdots x_{n_{k}}^{i_{k}}
$$

For a composition $I=\left(i_{1}, \ldots, i_{k}\right)$ we write $\ell(I)=k$ for the number of parts of $I$, and $|I|=i_{1}+\cdots+i_{k}$ for the sum of the parts of $I$. If $|I|=n$, we say $I$ is a composition of $n$ and write $I \vDash n$. If $I$ is a composition, there is a partition $\pi(I)$ given by forgetting the ordering: the monomial symmetric function $m_{\lambda}$ corresponding to a partition $\lambda$ is given by

$$
m_{\lambda}=\sum_{\pi(I)=\lambda} M_{I}
$$

and the monomial symmetric functions generate Sym as a vector space. For a partition $\lambda$, we use the notations $\ell(\lambda)$ and $|\lambda|$ in the same way as for compositions; if $|\lambda|=n$ we say $\lambda$ is a partition of $n$ and write $\lambda \vdash n$.

For a composition $\left(i_{1}, i_{2}, \ldots, i_{k}\right)$ with $i_{1}>1$, the corresponding multiple zeta value is the $k$-fold infinite series

$$
\zeta\left(i_{1}, i_{2}, \ldots, i_{k}\right)=\sum_{n_{1}>n_{2}>\cdots>n_{k} \geq 1} \frac{1}{n_{1}^{i_{1}} n_{2}^{i_{2}} \cdots n_{k}^{i_{k}}} .
$$

Multiple zeta values were introduced in [12] and [25], but the case $k=2$ actually goes back to Euler [7]. They have been studied extensively in recent decades, and have appeared in a surprising number of contexts, including knot theory and particle physics. Surveys include $[4,5,15]$.

The multiple zeta value (3) can be obtained from the monomial quasi-symmetric function $M_{\left(i_{k}, i_{k-1}, \ldots, i_{1}\right)}$ by sending $x_{n}$ to $\frac{1}{n}$, but the series won't converge unless $i_{1}>1$. If we let QSym $^{0}$ be the subspace of QSym generated by the monomial quasi-symmetric functions $M_{I}$ with the last part of $I$ greater than 1 , then it turns out that QSym ${ }^{0}$ is a subalgebra of QSym, and we have a homomorphism $\zeta: \mathrm{QSym}^{0} \rightarrow \mathbf{R}$ whose images are the multiple zeta values.

In fact (as we explain in the next section) QSym $=\operatorname{QSym}^{0}\left[M_{(1)}\right]$, so to extend $\zeta$ to a homomorphism defined on all of QSym it suffices to define $\zeta\left(M_{(1)}\right)$. As the author noted in [13], setting $\zeta\left(M_{(1)}\right)=\gamma$ (the Euler-Mascheroni constant) is a fruitful choice. If

$$
H(t)=1+h_{1} t+h_{2} t^{2}+\cdots \in \operatorname{Sym}[[t]]
$$

is the generating function for the complete symmetric functions

$$
h_{n}=\sum_{I \models n} M_{I}=\sum_{\lambda \vdash n} m_{\lambda},
$$


then setting $\zeta\left(M_{(1)}\right)=\gamma$ implies [13, Theorem 5.1]

$$
\zeta(H(t))=\Gamma(1-t)
$$

where $\Gamma$ is the usual gamma function. This is equivalent to

$$
\zeta(E(t))=\frac{1}{\Gamma(1+t)}
$$

where

$$
E(t)=1+e_{1} t+e_{2} t^{2}+\cdots
$$

is the generating function of the elementary symmetric functions $e_{j}=M_{\left(1^{j}\right)}$ (we write $1^{j}$ for a string of $j 1$ 's). The identity (4) was a key step in the proof in [13] that

$$
\sum_{n, m \geq 1} \zeta\left(n+1,1^{m-1}\right) s^{n} t^{m}=1-\exp \left(\sum_{i \geq 2} \zeta(i) \frac{t^{i}+s^{i}-(t+s)^{i}}{i}\right) .
$$

This latter identity (proved by a different method in [3]) is interesting since it shows that any multiple zeta value of the form $\zeta(n+1,1, \ldots, 1)$ can be expressed as a polynomial with rational coefficients in the ordinary zeta values $\zeta(i)$.

Libgober [17] showed that the $\Gamma$-genus appears in formulas that relate Chern classes of certain manifolds to the periods of their mirrors. The $\Gamma$-genus is the Hirzebruch [11] genus associated with the power series $Q(x)=\Gamma(1+x)^{-1}$, i.e., the genus coming from the multiplicative sequence of polynomials $\left\{Q_{i}\left(c_{1}, \ldots, c_{i}\right)\right\}$ in Chern classes, where

$$
\sum_{i=0}^{\infty} Q_{i}\left(e_{1}, \ldots, e_{i}\right)=\prod_{i=1}^{\infty} \frac{1}{\Gamma\left(1+x_{i}\right)}
$$

As shown in [14], the coefficient of the monomial $c_{\lambda}=c_{\lambda_{1}} c_{\lambda_{2}} \cdots$ in $Q_{i}\left(c_{1}, \ldots, c_{i}\right)$ is $\zeta\left(m_{\lambda}\right)$, for any partition $\lambda$. For example, using the tables in the Appendix, we have

$$
Q_{3}\left(c_{1}, c_{2}, c_{3}\right)=\zeta(3) c_{3}+(\gamma \zeta(2)-\zeta(3)) c_{1} c_{2}+\frac{1}{6}\left(\gamma^{3}-3 \gamma \zeta(2)+2 \zeta(3)\right) c_{1}^{3}
$$

More recently $\mathrm{Lu}[19]$ defined a similar $\hat{\Gamma}$-genus $\left\{P_{i}\right\}$ by using the generating function $P(x)=e^{-\gamma x} \Gamma(1+x)^{-1}$ in place of $Q(x)=\Gamma(1+x)^{-1}$, and related this new genus to an $S^{1}$-equivariant Euler class. The coefficient of $c_{\lambda}$ in $P_{i}\left(c_{1}, \ldots, c_{i}\right)$ can be obtained by setting $\gamma=0$ in $\zeta\left(m_{\lambda}\right)$. Thus

$$
P_{3}\left(c_{1}, c_{2}, c_{3}\right)=\zeta(3) c_{3}-\zeta(3) c_{1} c_{2}+\frac{1}{3} \zeta(3) c_{1}^{3}
$$

(cf. Table 1 of [19]). If we write $\hat{\zeta}$ for the function on QSym that sends $M_{(1)}$ to zero and agrees with $\zeta$ on QSym ${ }^{0}$, then

$$
\hat{\zeta}(E(t))=\frac{1}{e^{\gamma t} \Gamma(1+t)}
$$


Following the proof of the result of [14], we then have

$$
\sum_{i=0}^{\infty} P_{i}\left(e_{1}, \ldots, e_{i}\right) t^{i}=\prod_{i=1}^{\infty} \frac{1}{e^{\gamma x_{i} t} \Gamma\left(1+x_{i} t\right)}=\sum_{\lambda} \hat{\zeta}\left(e_{\lambda}\right) m_{\lambda} t^{|\lambda|}=\sum_{\lambda} \hat{\zeta}\left(m_{\lambda}\right) e_{\lambda} t^{|\lambda|}
$$

While equation (6) appears in [19] (see Prop. 4.3), it has a nice corollary that doesn't. Recall [11, Theorem 4.10.2] that the Chern classes of the tangent bundle of projective space $\mathbf{C P}^{n}$ are given by

$$
c_{i}=\left(\begin{array}{c}
n+1 \\
i
\end{array}\right) a^{i}
$$

with $a \in H^{2}\left(\mathbf{C P}^{n} ; \mathbf{Z}\right)$ such that $\left\langle a^{n},\left[\mathbf{C P}^{n}\right]\right\rangle=1$, where $\left[\mathbf{C P}^{n}\right] \in H_{2 n}\left(\mathbf{C P}^{n} ; \mathbf{Z}\right)$ is the fundamental class. Now by $[20$, p. 26] the specialization

$$
x_{i}= \begin{cases}1, & i=1,2, \ldots, n+1 \\ 0, & i>n+1\end{cases}
$$

sends $e_{i}$ to $\left(\begin{array}{c}n+1 \\ i\end{array}\right)$. It then follows from equation (6) that

$$
\hat{\Gamma}\left(\mathbf{C P}^{n}\right)=\left\langle P_{n}\left(c_{1}, \ldots, c_{n}\right),\left[\mathbf{C P}^{n}\right]\right\rangle=\text { coefficient of } t^{n} \text { in } \frac{1}{\left(e^{\gamma t} \Gamma(1+t)\right)^{n+1}}
$$

(cf. Table 2 of [19]).

As another occurrence of $\zeta$, we cite the following result about values of the derivatives of the gamma function at positive integers from [22]: if $n$ and $k$ are positive integers, then

$$
\frac{\Gamma^{(k)}(n)}{k !}=\sum_{j=0}^{k}\left[\begin{array}{c}
n \\
k+1-j
\end{array}\right](-1)^{j} \zeta\left(h_{j}\right),
$$

where $\left[\begin{array}{l}n \\ j\end{array}\right]$ is the number of permutations of degree $n$ with exactly $j$ cycles (Stirling number of the first kind). Cf. [23, pp. 40-44].

These examples suggest that the homomorphism $\zeta:$ QSym $\rightarrow \mathbf{R}$ may be useful to calculate. Now QSym is actually a Hopf algebra, as we discuss in the next section. Aguiar, Bergeron and Sottille [1] develop a theory of graded connected Hopf algebras endowed with characters (scalar-valued homomorphisms), in which "even" and "odd" characters are defined. A key result is that any such character $\chi$ is uniquely expressible as the convolution product $\chi_{+} \chi_{-}$of an even character $\chi_{+}$times an odd one $\chi_{-}$. In this paper we discuss some results on the character $\zeta$ : QSym $\rightarrow \mathbf{R}$ and its factors $\zeta_{+}$and $\zeta_{-}$, and particularly on the restrictions of these characters to Sym $\subset$ QSym. (Note that for the computation of the $\Gamma$ - and $\hat{\Gamma}$-genera, the restriction of $\zeta$ to Sym suffices.)

After developing some properties of the Hopf algebras QSym and Sym in $\S 2$, we discuss the factorization $\zeta=\zeta_{+} \zeta_{-}$on the full algebra QSym in $\S 3$. In $\S 4$ we consider the restriction of $\zeta, \zeta_{+}$and $\zeta_{-}$to Sym. First we show how to use the character table of the symmetric 
group to compute $\zeta$ on Schur functions. Then we consider the effect of $\zeta$ on the elementary and complete symmetric functions. We show equation (4) splits as

$$
\zeta_{+}(H(t))=\sqrt{\frac{\pi t}{\sin \pi t}} \text { and } \quad \zeta_{-}(H(t))=\Gamma(1-t) \sqrt{\frac{\sin \pi t}{\pi t}},
$$

which makes it easier to compute $\zeta$ on elementary and complete symmetric functions by computing $\zeta_{+}$and $\zeta_{-}$separately. Next we consider the values of the three characters on the monomial symmetric functions $m_{\lambda}$. While there is an explicit formula for $m_{\lambda}$ in terms of the $p_{\lambda}$ (Theorem 7 below), it is somewhat ineffective computationally since it involves a sum over set partitions. We develop some further methods by which the values of $\zeta$, $\zeta_{+}$, and $\zeta_{-}$can computed on $m_{\lambda}$, including an efficient algorithm for the case where $\lambda$ is a hook partition, i.e., $\lambda$ has at most one part greater than 1 (see equations (33) and (34) below). Finally, we discuss a family of symmetric functions in the kernel of $\zeta_{-}$. Values of $\zeta, \zeta_{+}$, and $\zeta_{-}$on $m_{\lambda}$ for $|\lambda| \leq 7$ are listed in the Appendix.

\section{The Hopf Algebras QSym and Sym}

As noted above, the monomial quasi-symmetric functions $M_{I}$ generate QSym as a vector space. The multiplication of the $M_{I}$ is given by a "quasi-shuffle" product, which involves combining parts of the associated compositions as well as shuffling them. For example,

$$
\begin{array}{r}
M_{(1)} M_{\left(i_{1}, i_{2}, \ldots, i_{l}\right)}=M_{\left(1, i_{1}, \ldots, i_{l}\right)}+M_{\left(i_{1}+1, i_{2}, \ldots, i_{l}\right)}+M_{\left(i_{1}, 1, i_{2}, \ldots, i_{l}\right)}+\cdots+ \\
M_{\left(i_{1}, i_{2}, \ldots, i_{l-1}, i_{l}+1\right)}+M_{\left(i_{1}, i_{2}, \ldots, i_{l}, 1\right)} .
\end{array}
$$

In fact, QSym is a polynomial algebra, as shown by by by Malvenuto and Reutenauer [21]. To state their result, we first define what it means for a composition $I$ to be Lyndon. If we order the compositions lexicographically, i.e.,

$$
(1)<(1,1)<(1,1,1)<\cdots<(1,2)<\cdots<(2)<(2,1)<\cdots<(3)<\cdots,
$$

then a composition $I$ is called Lyndon if $I<K$ for any nontrivial decomposition $I=J K$ of $I$ as a juxtaposition of shorter compositions. For example, $(1)$ and $(1,2,2)$ are Lyndon, but $(2,1)$ is not. Then the result of $[21]$ as follows.

Theorem 1. QSym is the polynomial algebra on the set $\left\{M_{I}: I\right.$ Lyndon $\}$.

The only Lyndon composition ending in 1 is (1) itself, so $\mathrm{QSym}^{0}$ is the subalgebra of QSym generated by the set $\left\{M_{I}: I\right.$ Lyndon, $\left.I \neq(1)\right\}$. Thus QSym $=\operatorname{QSym}^{0}\left[M_{(1)}\right]$, and we can be more specific as follows.

Theorem 2. Each monomial quasi-symmetric function $M_{I}$ can be expressed as a polynomial in $M_{(1)}$ with coefficients in QSym ${ }^{0}$, of degree equal to the number of trailing 1 's in $I$. 
Proof. Let $t(I)$ be the number of trailing 1's in $I$. Suppose the result holds for $M_{J}$ with $t(J) \leq n$, and consider $M_{I}$ with $t(I)=n+1$. Writing $I$ as the juxtaposition $I^{\prime}(1)$, it follows from equation $(7)$ with $\left(i_{1}, \ldots, i_{l}\right)=I^{\prime}$ that

$$
M_{(1)} M_{I^{\prime}}=\sum_{k=1}^{2 \ell\left(I^{\prime}\right)-n} M_{J_{k}}+(n+1) M_{I}
$$

where each $J_{k}$ has $t\left(J_{k}\right) \leq n$, so the result follows.

Now QSym is a graded connected Hopf algebra. If we adopt the convention that $M_{\emptyset}=1$, then the grade- $n$ part of QSym is generated by $\left\{M_{I}:|I|=n\right\}$. The counit $\epsilon$ is given by

$$
\epsilon\left(M_{I}\right)= \begin{cases}1, & \text { if } I=\emptyset \\ 0, & \text { otherwise }\end{cases}
$$

and coproduct $\Delta$ by

$$
\Delta\left(M_{I}\right)=\sum_{J K=I} M_{J} \otimes M_{K}
$$

It follows immediately from equation (8) that the only $M_{I}$ which are primitives are the power sums $p_{n}=M_{(n)}$.

The antipode $S:$ QSym $\rightarrow$ QSym is given by (see [6, Prop. 3.4])

$$
S\left(M_{I}\right)=(-1)^{\ell(I)} \sum_{\bar{I} \succeq J} M_{J},
$$

where $\bar{I}$ is the reverse of $I$ and $I \succeq J$ means $I$ is a refinement of $J$, i.e., $J$ is obtainable by combining some parts of $I$. Since QSym is commutative, $S$ is an automorphism of QSym with $S^{2}=$ id.

The algebra Sym is generated by the elementary symmetric functions $e_{n}$, and also by the complete symmetric functions $h_{n}$. The generating functions $E(t)$ and $H(t)$ for these symmetric functions are related by $E(t)=H(-t)^{-1}$. The power-sums $p_{n}$ also generate Sym as an algebra, and have generating function

$$
P(t)=p_{1}+p_{2} t+p_{3} t^{2}+\cdots=\frac{H^{\prime}(t)}{H(t)} .
$$

Now Sym is a sub-Hopf-algebra of QSym, and its structure is described succinctly by Geissinger [8]. As follows from equation $(9), S\left(e_{n}\right)=(-1)^{n} h_{n}$. The power-sums $p_{n}$ are primitive, and both the $e_{n}$ and $h_{n}$ are divided powers, i.e.,

$$
\Delta\left(e_{n}\right)=\sum_{i+j=n} e_{i} \otimes e_{j}
$$

and similarly for $h_{n}$. Stated in terms of generating functions, we have

$$
\Delta(E(t))=E(t) \otimes E(t) \quad \text { and } \quad \Delta(H(t))=H(t) \otimes H(t) .
$$


as well as

$$
\Delta(P(t))=P(t) \otimes 1+1 \otimes P(t) .
$$

As a vector space, Sym has the basis $\left\{m_{\lambda}: \lambda \in \Pi\right\}$, where $\Pi$ is the set of partitions. We also have bases

$$
\left\{e_{\lambda}: \lambda \in \Pi\right\}, \quad\left\{h_{\lambda}: \lambda \in \Pi\right\}, \quad \text { and } \quad\left\{p_{\lambda}: \lambda \in \Pi\right\}
$$

where $e_{\lambda}=e_{\lambda_{1}} e_{\lambda_{2}} \cdots e_{\lambda_{l}}$ for $\lambda=\left(\lambda_{1}, \lambda_{2}, \ldots, \lambda_{l}\right)$, and similarly for $h_{\lambda}$ and $p_{\lambda}$. Another important basis for Sym is the Schur functions $\left\{s_{\lambda}: \lambda \in \Pi\right\}$ (see $[20, \mathrm{I}, \S 3]$ ). For $\lambda=$ $\left(\lambda_{1}, \lambda_{2}, \ldots, \lambda_{l}\right)$ with $\lambda_{1} \geq \lambda_{2} \geq \cdots$, the corresponding Schur function $s_{\lambda}$ is the determinant

$$
\left|\begin{array}{cccc}
h_{\lambda_{1}} & h_{\lambda_{1}+1} & \cdots & h_{\lambda_{1}+l-1} \\
h_{\lambda_{2}-1} & h_{\lambda_{2}} & \cdots & h_{\lambda_{2}+l-2} \\
\vdots & \vdots & \ddots & \vdots \\
h_{\lambda_{l}-l+1} & h_{\lambda_{l}-l+2} & \cdots & h_{\lambda_{l}}
\end{array}\right|,
$$

where $h_{i}$ is interpreted as 1 if $i=0$ and 0 if $i<0$. Then $s_{(n)}=h_{n}$ and $s_{\left(1^{n}\right)}=e_{n}$.

There is an inner product on Sym defined by

$$
\left\langle h_{\mu}, m_{\lambda}\right\rangle=\delta_{\mu, \lambda}
$$

for all $\mu, \lambda \in \Pi$. As shown in [20, I, $\S 4]$, this inner product is symmetric and positive definite. The Schur functions are an orthonormal basis with respect to it, i.e.,

$$
\left\langle s_{\mu}, s_{\lambda}\right\rangle=\delta_{\mu, \lambda}
$$

For any symmetric function $f$ we can define its adjoint $f^{\perp}$ by

$$
\left\langle f^{\perp} u, v\right\rangle=\langle u, f v\rangle \text {. }
$$

For later use we recall from $[20$, p. 76$]$ that

$$
p_{r}^{\perp}=r \frac{\partial}{\partial p_{r}} .
$$

\section{The character $\zeta$ and its factors $\zeta_{+}$and $\zeta_{-}$}

Define $\zeta:$ QSym $\rightarrow \mathbf{R}$ by $\zeta(1)=1$,

$$
\zeta\left(M_{\left(i_{1}, \ldots, i_{k}\right)}\right)=\zeta\left(i_{k}, i_{k-1}, \ldots, i_{1}\right)
$$

for $i_{k}>1$, and $\zeta\left(M_{(1)}\right)=\gamma$. It follows from Theorem 2 that $\zeta\left(M_{I}\right)$ can be expressed as a polynomial in $\gamma$ with coefficients in the multiple zeta values, of degree equal to the number of trailing 1 's of $I$.

Following [1], we say a character of QSym (i.e., an algebra homomorphism $\chi:$ QSym $\rightarrow$ $\mathbf{R})$ is even if $\chi(u)=(-1)^{|u|} \chi(u)$ for homogeneous elements $u$, and odd if $\chi(u)=$ $(-1)^{|u|} \chi(S(u))$ for all homogeneous $u$. From [1] we have the following result. 
Theorem 3. For any character $\chi$ of QSym, there is a unique even character $\chi_{+}$and a unique odd character $\chi_{-}$so that $\chi$ is the convolution product $\chi_{+} \chi_{-}$.

From the preceding theorem, there are unique characters $\zeta_{-}$and $\zeta_{+}$of QSym so that $\zeta_{+}$is even, $\zeta_{-}$is odd, and $\zeta=\zeta_{+} \zeta_{-}$, i.e.,

$$
\zeta(u)=\sum_{u} \zeta_{+}\left(u^{\prime}\right) \zeta_{-}\left(u^{\prime \prime}\right)
$$

for all elements $u$ of QSym, where

$$
\Delta(u)=\sum_{u} u^{\prime} \otimes u^{\prime \prime}
$$

Since $M_{(n)}=p_{n}$ is primitive, we have from equation (13)

$$
\zeta(n)=\zeta_{+}\left(p_{n}\right)+\zeta_{-}\left(p_{n}\right) .
$$

This gives us the following result.

Theorem 4. If $n$ is even, $\zeta_{+}\left(p_{n}\right)=\zeta(n)$ and $\zeta_{-}\left(p_{n}\right)=0$. If $n$ is odd, $\zeta_{+}\left(p_{n}\right)=0$ and $\zeta_{-}\left(p_{n}\right)=\zeta(n)($ or $\gamma$ if $n=1)$.

Proof. For even $n$, the oddness of $\zeta_{-}$implies

$$
\zeta_{-}\left(p_{n}\right)=\zeta_{-}\left(S\left(p_{n}\right)\right)=-\zeta_{-}\left(p_{n}\right)
$$

and the first statement follows from equation (14). If $n$ is odd, then $\zeta_{+}\left(p_{n}\right)=0$ and the second statement follows from equation (14).

The result that $\zeta_{-}\left(p_{n}\right)=0$ for $n$ even can be generalized as follows. Call a composition $I$ even if all its parts are even.

Theorem 5. If I is even, then $\zeta_{-}\left(M_{I}\right)=0$.

Proof. We make use of the universal character $\zeta_{Q}:$ QSym $\rightarrow \mathbf{R}$ given by

$$
\zeta_{Q}\left(M_{I}\right)= \begin{cases}1, & \text { if } \ell(I)=1 \\ 0, & \text { otherwise }\end{cases}
$$

By [1, Theorem 4.1], there is a unique homomorphism $\Psi:$ QSym $\rightarrow$ QSym such that $\zeta_{Q} \circ \Psi=\zeta$. Further, $\Psi$ is given by

$$
\Psi\left(M_{I}\right)=\sum_{I=I_{1} I_{2} \cdots I_{h}} \zeta\left(M_{I_{1}}\right) \zeta\left(M_{I_{2}}\right) \cdots \zeta\left(M_{I_{h}}\right) M_{\left(\left|I_{1}\right|, \ldots,\left|I_{h}\right|\right)},
$$

where the sum is over all decompositions of $I$ into a juxtaposition $I_{1} I_{2} \cdots I_{h}$ of compositions. Lemma 2.2 of [2] implies that $\zeta_{Q-} \circ \Psi=\zeta_{-}$, so

$$
\zeta_{-}\left(M_{I}\right)=\sum_{I=I_{1} I_{2} \cdots I_{h}} \zeta\left(M_{I_{1}}\right) \zeta\left(M_{I_{2}}\right) \cdots \zeta\left(M_{I_{h}}\right) \zeta_{Q-}\left(M_{\left(\left|I_{1}\right|, \ldots,\left|I_{h}\right|\right)}\right)
$$

Now an explicit formula for $\zeta_{Q-}\left(M_{J}\right)$ is given by [2, Theorem 3.2], which implies that $\zeta_{Q-}\left(M_{J}\right)=0$ whenever the last part of $J$ is even. Since $\left(\left|I_{1}\right|,\left|I_{2}\right|, \ldots,\left|I_{h}\right|\right)$ is even whenever $I$ is, the conclusion follows. 
It follows from the preceding result and equation (13) that $\zeta_{+}\left(M_{I}\right)=\zeta\left(M_{I}\right)$ for $I$ even. Nevertheless, for most compositions $I$ with $|I|$ even it is no easier to compute $\zeta_{+}\left(M_{I}\right)$ or $\zeta_{-}\left(M_{I}\right)$ than $\zeta\left(M_{I}\right)$. In fact, the bound on the degree of $\gamma$ in $\zeta\left(M_{I}\right)$ given by Theorem 2 need not hold for $\zeta_{+}\left(M_{I}\right)$ and $\zeta_{-}\left(M_{I}\right)$. For example,

$$
\zeta\left(M_{(1,2,3)}\right)=\zeta(3,2,1)=3 \zeta(3)^{2}-\frac{203}{48} \zeta(6),
$$

while

$$
\zeta_{+}\left(M_{(1,2,3)}\right)=-\gamma \zeta(2) \zeta(3)+\frac{11}{4} \gamma \zeta(5)+\frac{5}{2} \zeta(3)^{2}-\frac{203}{48} \zeta(6)
$$

and

$$
\zeta_{-}\left(M_{(1,2,3)}\right)=\gamma \zeta(2) \zeta(3)-\frac{11}{4} \gamma \zeta(5)+\frac{1}{2} \zeta(3)^{2}
$$

(note equation (13) gives $\zeta\left(M_{(1,2,3)}\right)=\zeta_{+}\left(M_{(1,2,3)}\right)+\zeta_{-}\left(M_{(1,2,3)}\right)$ here). As we see in the next section, the situation is dramatically different when these characters are restricted to Sym $\subset$ QSym.

\section{The restriction of $\zeta$ to Sym}

The vector space Sym has the various bases $m_{\lambda}, e_{\lambda}, h_{\lambda}, p_{\lambda}$ and $s_{\lambda}$ discussed in $\S 2$. We shall consider the last two bases first. We know the values of $\zeta$ in the basis elements $p_{\lambda}$ immediately from the definition, since

$$
\zeta\left(p_{i}\right)= \begin{cases}\gamma, & \text { if } i=1 \\ \zeta(i), & \text { otherwise }\end{cases}
$$

From Theorem 4 and Euler's identity for $\zeta(i), i$ even,

$$
\zeta_{+}\left(p_{i}\right)= \begin{cases}\frac{2^{i-1}\left|B_{i}\right|}{i !} \pi^{i}, & \text { if } i \text { is even, } \\ 0, & \text { otherwise }\end{cases}
$$

so it follows that $\zeta_{+}(u)$ for an element $u \in$ Sym of even degree $d$ is a rational multiple of $\pi^{d}$ (or alternatively of $\zeta(d)$ ). Of course $\zeta_{+}(u)=0$ if $u$ has odd degree. Also

$$
\zeta_{-}\left(p_{i}\right)= \begin{cases}\gamma, & \text { if } i=1 \\ \zeta(i), & \text { if } i>1 \text { is odd } \\ 0, & \text { otherwise }\end{cases}
$$

so the value $\zeta_{-}(u)$ on any $u \in$ Sym is a polynomial in $\gamma, \zeta(3), \zeta(5), \ldots$

Now the transition matrix from the $p_{\lambda}$ to the Schur functions $s_{\lambda}$ is provided by the character table of the symmetric group $S_{n}$ (see $[20, \mathrm{I}, \S 7]$ ). The irreducible characters of $S_{n}$ are indexed by the partitions of $n$ : let $\chi^{\lambda}$ be the character associated with $\lambda$. The 
value $\chi^{\lambda}(\sigma)$ of the character $\chi^{\lambda}$ on a permutation $\sigma \in S_{n}$ only depends on the conjugacy class of $\sigma$, i.e., its cycle-type: the cycle-type corresponding to the partition $\rho \vdash n$ is

$$
\left\{\sigma \in S_{n}: \sigma \text { has } m_{i}(\rho) i \text {-cycles for } 1 \leq i \leq n\right\}
$$

where $m_{i}(\rho)$ is the number of parts of $\rho$ equal to $i$. If we let $\chi_{\rho}^{\lambda}=\chi^{\lambda}(\sigma)$ for $\sigma$ of cycle-type $\rho$, the numbers $\chi_{\rho}^{\lambda}$ completely determine the character $\chi^{\lambda}$. From [20] we have the following result.

Proposition. For any partition $\lambda$ of $n$,

$$
s_{\lambda}=\sum_{\rho \vdash n} \frac{\chi_{\rho}^{\lambda}}{z_{\rho}} p_{\rho},
$$

where

$$
z_{\rho}=m_{1}(\rho) ! m_{2}(\rho) ! 2^{m_{2}(\rho)} m_{3}(\rho) ! 3^{m_{3}(\rho)} \cdots
$$

Two special cases are worth noting: $\lambda=(n)$ and $\lambda=\left(1^{n}\right)$. In the first case $\chi^{(n)}$ is the trivial character, and equation (15) is

$$
h_{n}=\sum_{i_{1}+2 i_{2}+\cdots=n} \frac{1}{i_{1} ! 1^{i_{1}} i_{2} ! 2^{i_{2}} \cdots i_{n} ! n^{i_{n}}} p_{1}^{i_{1}} p_{2}^{i_{2}} \cdots p_{n}^{i_{n}} .
$$

In the second, $\chi^{\left(1^{n}\right)}$ is the alternating character of $S_{n}$, i.e.,

$$
\chi^{\left(1^{n}\right)}(\sigma)=\operatorname{sign} \text { of } \sigma=(-1)^{m_{2}(\rho)+m_{4}(\rho)+\cdots}
$$

where $\rho$ is the cycle-type of $\sigma$. In this case equation (15) becomes

$$
e_{n}=\sum_{i_{1}+2 i_{2}+\cdots=n} \frac{(-1)^{i_{2}+i_{4}+\cdots}}{i_{1} ! 1^{i_{1}} i_{2} ! 2^{i_{2}} \cdots i_{n} ! n^{i_{n}}} p_{1}^{i_{1}} p_{2}^{i_{2}} \cdots p_{n}^{i_{n}} .
$$

At this point we could compute $\zeta$ on the bases $h_{\lambda}$ and $e_{\lambda}$ by applying $\zeta$ to equations (16) and (17) respectively (cf. [10, Prop. 2]). But as we shall see shortly, it is much more efficient to split $\zeta$ into even and odd parts.

Applying $\zeta$ to equation (15), we obtain

$$
\zeta\left(s_{\lambda}\right)=\sum_{\rho \vdash n} \frac{\chi_{\rho}^{\lambda}}{z_{\rho}} \gamma^{m_{1}(\rho)} \zeta(2)^{m_{2}(\rho)} \zeta(3)^{m_{3}(\rho)} \cdots,
$$

which can be written in the alternative form

$$
\zeta\left(s_{\lambda}\right)=\sum_{\rho \vdash n} \frac{\chi_{\rho}^{\lambda} N(\rho)}{n !} \gamma^{m_{1}(\rho)} \zeta(2)^{m_{2}(\rho)} \zeta(3)^{m_{3}(\rho)} \ldots,
$$


where $N(\rho)$ is the number of permutations of cycle-type $\rho$. For example, using the tables of group characters in [18], equation (18) gives

$$
\begin{aligned}
\zeta\left(s_{(3,2,1)}\right) & =\frac{16}{6 !} \gamma^{6}-\frac{2 \cdot 40}{6 !} \gamma^{3} \zeta(3)+\frac{144}{6 !} \gamma \zeta(5)-\frac{2 \cdot 40}{6 !} \zeta(3)^{2} \\
& =\frac{1}{45} \gamma^{6}-\frac{1}{9} \gamma^{3} \zeta(3)+\frac{1}{5} \gamma \zeta(5)-\frac{1}{9} \zeta(3)^{2}
\end{aligned}
$$

Now we turn to the values of $\zeta$ on the bases $e_{\lambda}$ and $h_{\lambda}$. The two are closely related, because the automorphism $\omega$ of Sym defined by $\omega(u)=(-1)^{|u|} S(u)$ simply exchanges the two (see $[20, \mathrm{I}, \S 2])$. The values of $\zeta_{+}\left(e_{n}\right)$ and $\zeta_{+}\left(h_{n}\right)$ are given by the following result.

\section{Theorem 6.}

$$
\zeta_{+}(H(t))=\sqrt{\frac{\pi t}{\sin \pi t}} \text { and } \zeta_{+}(E(t))=\sqrt{\frac{\sin \pi t}{\pi t}} .
$$

Proof. The oddness of $\zeta_{-}$means it is $\omega$-invariant, so $\omega(E(t))=H(t)$ implies

$$
\zeta_{-}(H(t))=\zeta_{-}(E(t))
$$

Since $\zeta_{+}(H(t))$ is an even function of $t$,

$$
\zeta_{+}(H(t))=\zeta_{+}(H(-t))=\zeta_{+}\left(E(t)^{-1}\right)=\zeta_{+}(E(t))^{-1}
$$

Now using equations (4) and (5) together with (10), we have

$$
\begin{aligned}
\Gamma(1-t) \Gamma(1+t) & =\zeta(H(t)) \zeta(E(t))^{-1} \\
& =\zeta_{+}(H(t)) \zeta_{-}(H(t)) \zeta_{+}(E(t))^{-1} \zeta_{-}(E(t))^{-1}
\end{aligned}
$$

and from equations (19) and (20) the right-hand side simplifies to $\zeta_{+}(H(t))^{2}$. Using the reflection formula for the gamma function and taking square roots, we have

$$
\zeta_{+}(H(t))=\sqrt{\frac{\pi t}{\sin \pi t}}
$$

and thus, by equation (20), the conclusion.

From the preceding result, the $\zeta_{-}\left(e_{n}\right)$ are given by

$$
\zeta_{-}(E(t))=\zeta_{-}(H(t))=\frac{\zeta(H(t))}{\zeta_{+}(H(t))}=\Gamma(1-t) \sqrt{\frac{\sin \pi t}{\pi t}} .
$$

We can also apply $\zeta_{-}$to both sides of equation (17) to obtain

$$
\zeta_{-}\left(e_{n}\right)=\sum_{i_{1}+3 i_{3}+5 i_{5} \ldots=n} \frac{\gamma^{i_{1}} \zeta(3)^{i_{3}} \zeta(5)^{i_{5}} \cdots}{i_{1} ! 1^{i_{1}} i_{3} ! 3^{i_{3}} i_{5} ! 5^{i_{5}} \ldots}
$$

for all positive integers $n$. 
Since the $e_{n}$ are divided powers,

$$
\zeta\left(e_{n}\right)=\sum_{i+j=n} \zeta_{+}\left(e_{i}\right) \zeta_{-}\left(e_{j}\right)
$$

and we need only consider those terms in the sum (22) with $i$ even. So from

$$
\sqrt{\frac{\sin \pi t}{\pi t}}=1-\frac{\pi^{2} t}{12}+\frac{\pi^{4} t^{4}}{1440}-\frac{\pi^{6} t^{6}}{24192}+\cdots
$$

we can compute $\zeta\left(e_{6}\right)$ using equations $(21)$ and $(22)$ as

$$
\begin{aligned}
& \zeta_{-}\left(e_{6}\right)+\zeta_{+}\left(e_{2}\right) \zeta_{-}\left(e_{4}\right)+\zeta_{+}\left(e_{4}\right) \zeta_{-}\left(e_{2}\right)+\zeta_{+}\left(e_{6}\right)= \\
& \frac{\gamma^{6}}{720}+\frac{\gamma^{3} \zeta(3)}{18}+\frac{\gamma \zeta(5)}{5}+\frac{\zeta(3)^{2}}{18}-\frac{\pi^{2}}{12}\left(\frac{\gamma^{4}}{24}+\frac{\gamma \zeta(3)}{3}\right)+\frac{\pi^{4}}{1440} \frac{\gamma^{2}}{2}-\frac{\pi^{6}}{24192} \\
& \quad=\frac{\gamma^{6}}{720}-\frac{\gamma^{4} \pi^{2}}{288}+\frac{\gamma^{3} \zeta(3)}{18}+\frac{\gamma^{2} \pi^{4}}{2880}+\gamma\left(\frac{\zeta(5)}{5}-\frac{\pi^{2} \zeta(3)}{36}\right)+\frac{\zeta(3)^{2}}{18}-\frac{\pi^{6}}{24192}
\end{aligned}
$$

The $h_{n}$ are also divided powers, so we can compute $\zeta\left(h_{n}\right)$ similarly, using Theorem 6 and equation $(21)$ (since $\zeta_{-}\left(e_{n}\right)=\zeta_{-}\left(h_{n}\right)$ ).

Finally, we consider the basis $m_{\lambda}$. Since the power-sums $p_{i}$ generate Sym over the rational numbers, there exists for each partition $\lambda$ a polynomial $P_{\lambda}$ (with rational coefficients) so that

$$
m_{\lambda}=P_{\lambda}\left(p_{1}, p_{2}, \ldots\right)
$$

From Theorem 4 we then have

$$
\zeta_{+}\left(m_{\lambda}\right)=P_{\lambda}(0, \zeta(2), 0, \zeta(4), 0, \ldots)
$$

and

$$
\zeta_{-}\left(m_{\lambda}\right)=P_{\lambda}(\gamma, 0, \zeta(3), 0, \zeta(5), 0, \ldots),
$$

since $\zeta_{+}$and $\zeta_{-}$are homomorphisms. (Of course, $\zeta_{+}\left(m_{\lambda}\right)=0$ if $|\lambda|$ is odd.) Once $\zeta_{+}$and $\zeta_{-}$are known on the monomial basis, the values of $\zeta$ can be computed using the fact [8] that

$$
\Delta\left(m_{\lambda}\right)=\sum_{\alpha \cup \beta=\lambda} m_{\alpha} \otimes m_{\beta},
$$

where $\alpha \cup \beta$ means the union as multisets. Therefore

$$
\zeta\left(m_{\lambda}\right)=\sum_{\alpha \cup \beta=\lambda} \zeta_{+}\left(m_{\alpha}\right) \zeta_{-}\left(m_{\beta}\right)
$$

Note that we need only consider those terms in (25) with $|\alpha|$ even.

In fact the polynomials $P_{\lambda}$ have an explicit formula, which follows from [16, Theorem 2.3] (see also [12, Theorem 2.2]). We need some notation. If $\mathcal{B}=\left\{B_{1}, \ldots, B_{l}\right\}$ is a partition of the set $\{1,2, \ldots, k\}$, we write

$$
c(\mathcal{B})=(-1)^{k-l}\left(\operatorname{card} B_{1}-1\right) !\left(\operatorname{card} B_{2}-1\right) ! \cdots\left(\operatorname{card} B_{l}-1\right) ! .
$$

Then our formula is as follows. 
Theorem 7. For $\lambda=\left(\lambda_{1}, \lambda_{2}, \ldots, \lambda_{k}\right) \in \Pi$,

$$
m_{\lambda}=\frac{1}{m_{1}(\lambda) ! m_{2}(\lambda) ! \cdots} \sum_{\text {partitions } \mathcal{B}=\left\{B_{1}, \ldots, B_{l}\right\} \text { of }\{1, \ldots, k\}} c(\mathcal{B}) p_{b_{1}} p_{b_{2}} \cdots p_{b_{l}},
$$

where $b_{i}=\sum_{j \in B_{i}} \lambda_{j}$.

For example, taking partitions into two parts we have

$$
\begin{aligned}
& m_{(a, b)}=p_{a} p_{b}-p_{a+b} \\
& m_{(a, a)}=\frac{1}{2}\left(p_{a}^{2}-p_{2 a}\right)
\end{aligned}
$$

and taking those with three parts gives

$$
\begin{aligned}
& m_{(a, b, c)}=p_{a} p_{b} p_{c}-p_{a} p_{b+c}-p_{b} p_{a+c}-p_{c} p_{a+b}+2 p_{a+b+c} \\
& m_{(a, b, b)}=\frac{1}{2}\left(p_{a} p_{b}^{2}-2 p_{b} p_{a+b}-p_{a} p_{2 b}+2 p_{a+2 b}\right) \\
& m_{(a, a, a)}=\frac{1}{6}\left(p_{a}^{3}-3 p_{a} p_{2 a}+2 p_{3 a}\right)
\end{aligned}
$$

for $a, b, c$ distinct.

The characters $\zeta, \zeta_{+}, \zeta_{-}$can be computed on any $m_{\lambda}$ by applying them to Theorem 7 . But since it involves a sum over set partitions, the theorem is less effective computationally than it appears. Nevertheless, for some partitions $\lambda$ the sum in Theorem 7 reduces to a sum over integer partitions. With a little work, equation (17) can be derived from Theorem 7 with $\lambda=\left(1^{n}\right)$. We also have the following result on hook partitions $\lambda=\left(n, 1^{t}\right)$.

Corollary. If $n>1$, then

$$
m_{\left(n, 1^{t}\right)}=\sum_{j=0}^{t}(-1)^{j} p_{n+j} e_{t-j}
$$

Proof. Let $\lambda=\left(n, 1^{t}\right)$, and consider a partition

$$
\mathcal{B}=\left\{B_{1}, \ldots, B_{l}\right\} \quad \text { of } \quad\{1,2, \ldots, t+1\} .
$$

We order the blocks $B_{i}$ so that $B_{1}$ always includes 1 . The $b_{i}$ as in the conclusion of Theorem 7 are $b_{1}=n+\operatorname{card} B_{1}-1$, and $b_{i}=$ card $B_{i}$ for $i>1$. The sets $B_{1}-\{1\}, B_{2}, \ldots, B_{l}$ form a partition of $\{2, \ldots, t+1\}$ : let $c_{1}, \ldots, c_{l}$ be their respective cardinalities. Then

$$
c(\mathcal{B})=(-1)^{t+1-l} c_{1} !\left(c_{2}-1\right) ! \cdots\left(c_{l}-1\right) ! .
$$

The number of distinct partitions of $\{2, \ldots, t+1\}$ corresponding to given values of $c_{1}, c_{2}, \ldots, c_{l}$ is

$$
\left(\begin{array}{ccc}
t & \\
c_{1} c_{2} & \cdots & c_{l}
\end{array}\right) \frac{1}{i_{1} ! i_{2} ! \cdots}
$$


where $i_{j}=\operatorname{card}\left\{m \geq 2: c_{m}=j\right\}$. The factors (31) and (32) have product

$$
(-1)^{t-(l-1)} \frac{t !}{c_{2} c_{3} \cdots c_{l}} \frac{1}{i_{1} ! i_{2} ! \cdots}=(-1)^{t+i_{1}+i_{2}+\cdots} \frac{t !}{i_{1} ! 1^{i_{1}} i_{2} ! 2^{i_{2}} \cdots},
$$

and from Theorem 7 it follows that

$$
m_{\left(n, 1^{t}\right)}=\sum_{i_{1}+2 i_{2}+\cdots \leq t} \frac{(-1)^{t+i_{1}+i_{2}+\cdots}}{i_{1} ! 1^{i_{1}} i_{2} ! 2^{i_{2}} \cdots} p_{n+t-i_{1}-2 i_{2}-\cdots} p_{1}^{i_{1}} p_{2}^{i_{2}} \cdots
$$

Now apply equation (17) to obtain the conclusion.

Applying $\zeta_{+}$and $\zeta_{-}$to both sides of the preceding result,

$$
\zeta_{+}\left(m_{\left(n, 1^{t}\right)}\right)=(-1)^{t} \sum_{i=0}^{t} \zeta_{+}\left(p_{n+i}\right) \zeta_{+}\left(e_{t-i}\right)
$$

and

$$
\zeta_{-}\left(m_{\left(n, 1^{t}\right)}\right)=(-1)^{n+1} \sum_{i=0}^{t} \zeta_{-}\left(p_{n+i}\right) \zeta_{-}\left(e_{t-i}\right),
$$

where in equation (33) we only include terms with $n+i$ even, and in (34) we only take terms with $n+i$ odd. To illustrate these formulas, we compute the values of $\zeta, \zeta_{+}$and $\zeta_{-}$on $m_{\left(5,1^{3}\right)}$. Equations (33) and (34) give respectively

$$
\zeta_{+}\left(m_{\left(5,1^{3}\right)}\right)=(-1)^{3}\left(\zeta(6) \zeta_{+}\left(e_{2}\right)+\zeta(8)\right)=-\frac{1}{6} \zeta(8)
$$

and

$$
\zeta_{-}\left(m_{\left(5,1^{3}\right)}\right)=(-1)^{6}\left(\zeta(5) \zeta_{-}\left(e_{3}\right)+\zeta(7) \zeta_{-}\left(e_{1}\right)\right)=\frac{1}{6} \gamma^{3} \zeta(5)+\gamma \zeta(7)+\frac{1}{3} \zeta(3) \zeta(5)
$$

Now apply equation (25) to get

$$
\begin{aligned}
\zeta\left(m_{\left(5,1^{3}\right)}\right) & =\zeta_{-}\left(m_{\left(5,1^{3}\right)}\right)+\zeta_{+}\left(e_{2}\right) \zeta_{-}\left(m_{(5,1)}\right)+\zeta_{+}\left(m_{(5,1)}\right) \zeta_{-}\left(e_{2}\right)+\zeta_{+}\left(m_{\left(5,1^{3}\right)}\right) \\
& =\frac{1}{6} \gamma^{3} \zeta(5)-\frac{1}{2} \gamma^{2} \zeta(6)+\gamma\left(\zeta(7)-\frac{1}{2} \zeta(2) \zeta(5)\right)+\frac{1}{3} \zeta(3) \zeta(5)-\frac{1}{6} \zeta(8) .
\end{aligned}
$$

A table of the polynomials $P_{\lambda}$ can also be built up by formal antidifferentiation, as we now explain.

Theorem 8. For any partitions $\lambda$ and $\mu$, define

$$
P_{\lambda-\mu}= \begin{cases}P_{\pi}, & \text { if } \lambda=\mu \cup \pi \\ 0, & \text { otherwise. }\end{cases}
$$

Then

$$
\frac{\partial P_{\lambda}}{\partial p_{r}}=\frac{1}{r} \sum_{\mu \vdash r} c_{\mu} P_{\lambda-\mu}
$$

where

$$
p_{r}=\sum_{\mu \vdash r} c_{\mu} h_{\mu}
$$


Proof. We recall the inner product $\langle\cdot, \cdot\rangle$ defined by (11). For all partitions $\pi$ we have

$$
\begin{aligned}
\left\langle\frac{\partial P_{\lambda}}{\partial p_{r}}\left(p_{1}, p_{2}, \ldots\right), h_{\pi}\right\rangle=\frac{1}{r}\left\langle p_{r}^{\perp} m_{\lambda}, h_{\pi}\right\rangle=\frac{1}{r}\left\langle m_{\lambda}, p_{r} h_{\pi}\right\rangle & = \\
\frac{1}{r} \sum_{\mu \vdash r} c_{\mu}\left\langle m_{\lambda}, h_{\mu \cup \pi}\right\rangle & =\left\langle\frac{1}{r} \sum_{\mu \vdash r} c_{\mu} P_{\lambda-\mu}\left(p_{1}, p_{2}, \ldots\right), h_{\pi}\right\rangle,
\end{aligned}
$$

where we have used equation (12).

Thus, from

$$
\begin{aligned}
& p_{1}=h_{1} \\
& p_{3}=h_{1}^{3}-3 h_{1} h_{2}+3 h_{3} \\
& p_{5}=h_{1}^{5}-5 h_{1}^{3} h_{2}+5 h_{1}^{2} h_{3}+5 h_{1} h_{2}^{2}-5 h_{1} h_{4}-5 h_{2} h_{3}+5 h_{5}
\end{aligned}
$$

it follows that

$$
\begin{aligned}
& \frac{\partial P_{\lambda}}{\partial p_{1}}=P_{\lambda-(1)} \\
& \frac{\partial P_{\lambda}}{\partial p_{3}}=\frac{1}{3} P_{\lambda-\left(1^{3}\right)}-P_{\lambda-(2,1)}+P_{\lambda-(3)} \\
& \frac{\partial P_{\lambda}}{\partial p_{5}}=\frac{1}{5} P_{\lambda-\left(1^{5}\right)}-P_{\lambda-\left(2,1^{3}\right)}+P_{\lambda-(3,1,1)}+P_{\lambda-(2,2,1)}-P_{\lambda-(4,1)}-P_{\lambda-(2,3)}+P_{\lambda-(5)} .
\end{aligned}
$$

Now to find, e.g., $\zeta_{-}\left(m_{(5,3,1,1)}\right)$ we begin with

$$
\zeta_{-}\left(m_{(5,3,1)}\right)=\gamma \zeta(3) \zeta(5)+2 \zeta(9),
$$

obtainable by applying $\zeta_{-}$to equation (28). From the preceding result with $r=1$,

$$
\zeta_{-}\left(m_{(5,3,1,1)}\right)=\frac{1}{2} \gamma^{2} \zeta(3) \zeta(5)+2 \gamma \zeta(9)+\alpha \zeta(3) \zeta(7)+\beta \zeta(5)^{2}
$$

for some rational numbers $\alpha, \beta$. Since

$$
\frac{\partial P_{(5,3,1,1)}}{\partial p_{3}}=P_{(5,1,1)}
$$

we have

$$
\frac{1}{2} \gamma^{2} \zeta(5)+\alpha \zeta(7)=\zeta_{-}\left(m_{(5,1,1)}\right)
$$

and comparing with $\zeta_{-}$applied to equation (29) (with $a=5$ and $b=1$ ) gives $\alpha=1$. But also

So

$$
\frac{\partial P_{(5,3,1,1)}}{\partial p_{5}}=P_{(3,1,1)}+P_{(5)}
$$

$$
\frac{1}{2} \gamma^{2} \zeta(3)+2 \beta \zeta(5)=\zeta_{-}\left(m_{(3,1,1)}\right)+\zeta(5)
$$


from which we see that $\beta=1$. Thus

$$
\zeta_{-}\left(m_{(5,3,1,1)}\right)=\frac{1}{2} \gamma^{2} \zeta(3) \zeta(5)+2 \gamma \zeta(9)+\zeta(3) \zeta(7)+\zeta(5)^{2}
$$

Another check on tables of $\zeta_{-}\left(m_{\lambda}\right)$ is provided by a series of identities that show certain symmetric functions are in the kernel of $\zeta_{-}$. For $\pi \in \Pi$, let $L(\pi)$ be the number of parts of $\pi$ of size greater than 1 . Set

$$
L_{n, k}=\sum_{|\pi|=n, L(\pi)=k} m_{\pi}
$$

Note that $L_{n, k}=0$ unless $k \leq\left\lfloor\frac{n}{2}\right\rfloor$. We define the "excess" of $L_{n, k}$ by $e\left(L_{n, k}\right)=n-2 k$, so the excess of a nonzero $L_{n, k}$ is always nonnegative.

Lemma. For integers $e \geq 1$ and $k \geq 0$,

$$
p_{1} L_{2 k+e-1, k}+p_{2} L_{2 k+e-2, k}+\cdots+p_{e} L_{2 k, k}=e L_{2 k+e, k}+2(k+1) L_{2 k+e, k+1} .
$$

Proof. First note that if we define $L(I)$ for a composition $I$ to be the number of parts of $I$ of size greater than 1 , then

$$
L_{n, k}=\sum_{|I|=n, L(I)=k} M_{I}
$$

Consider an individual monomial quasi-symmetric function in $L_{2 k+e, k}$, say $M_{(2,1,4,2,1)}$ in the case $k=3$ and $e=4$. It can arise in the sum

$$
M_{(1)} L_{2 k+e-1, k}+M_{(2)} L_{2 k+e-2, k}+\cdots+M_{(e)} L_{2 k, k}
$$

from $M_{(1)} M_{(2,4,2,1)}, M_{(1)} M_{(2,1,4,2)}, M_{(1)} M_{(2,1,3,2,1)}$, and $M_{(2)} M_{(2,1,2,2,1)}$. More generally, $M_{I}$ in $L_{2 k+e, k}$ arises in (35) in

$$
P_{1}(I)-P_{2}(I)+P_{3}(I)+P_{4}(I)+\cdots=|I|-2 P_{2}(I)
$$

ways, where $P_{r}(I)$ is the number of parts of $I$ of size $\geq r$. But in fact (36) is just the excess $e$ of $L_{2 k+e, k}$, thus establishing the coefficient $e$ in the lemma. Now consider a monomial quasi-symmetric function in $L_{2 k+e, k+1}$, say $M_{(2,3,2,3)}$ in the case $k=3, e=4$. It can arise in (35) from any of the eight terms

$$
\begin{aligned}
& M_{(1)} M_{(1,3,2,3)}, M_{(1)} M_{(2,2,2,3)}, M_{(1)} M_{(2,3,1,3)}, M_{(1)} M_{(2,3,2,2)}, \\
& M_{(2)} M_{(3,2,3)}, M_{(2)} M_{(2,3,3)}, M_{(3)} M_{(2,2,3)}, M_{(3)} M_{(2,3,2)} .
\end{aligned}
$$

In general, $M_{I}$ in $L_{2 k+e, k+1}$ arises in (35) in $2(k+1)$ ways, giving the coefficient $2(k+1)$.

Theorem 9. If $k \geq 1$, then $\zeta_{-}\left(L_{n, k}\right)=0$. 
Proof. We use induction on the excess of $L_{n, k}$. Since for $k \geq 1$

$$
\zeta_{-}\left(L_{2 k, k}\right)=\zeta_{-}\left(m_{(2,2, \ldots, 2)}\right)=0,
$$

the theorem evidently holds for excess 0 . Suppose it holds for excess $\leq n$. From the lemma we have

$$
L_{n+2 k+1, k}=\frac{1}{n+1}\left[p_{1} L_{n+2 k, k}+\cdots+p_{n+1} L_{2 k, k}-2(k+1) L_{n+2 k+1, k+1}\right],
$$

and every $L$ on the right-hand side has excess $n$ or less. Applying $\zeta_{-}$to both sides, it

follows from the induction hypothesis that the theorem also holds for $L_{n+2 k+1, k}$.

Remark. If $k=0$, then $\zeta_{-}\left(L_{n, k}\right)=\zeta_{-}\left(e_{n}\right)$ is given by equation (21).

\section{References}

[1] M. Aguiar, N. Bergeron, and F. Sottille, Combinatorial Hopf algebras and generalized Dehn-Somerville relations, Compos. Math. 142 (2006), 1-30.

[2] M. Aguiar and S. K. Hsiao, Canonical characters on quasi-symmetric functions and bivariate Catalan numbers, Electron. J. Combin. 11(2) (2004), Res. Art. 15.

[3] J. M. Borwein, D. M. Bradley, and D. J. Broadhurst, Evaluation of $k$-fold Euler/Zagier sums: a compendium of results for arbitrary $k$, Electron. J. Combin. 4(2) (1997), Res. Art. 5.

[4] J. M. Borwein, D. M. Bradley, D. J. Broadhurst, and P. Lisoněk, Special values of multidimensional polylogarithms, Trans. Amer. Math. Soc. 353 (2001), 907-941.

[5] D. Bowman and D. M. Bradley, Multiple polylogarithms: a brief survey, in q-Series with Applications to Combinatorics, Number Theory, and Physics, Contemp. Math., Vol. 291, American Mathematical Society, Providence, 2001, pp. 71-92.

[6] R. Ehrenborg, On posets and Hopf algebras, Adv. Math 119 (1996), 1-25.

[7] L. Euler, Meditationes circa singulare serierum genus, Novi Comm. Acad. Sci. Petropol. 20 (1775), 140-186; reprinted in Opera Omnia, Ser. I, Vol. 16(2), B. G. Teubner, Leipzig, 1935, pp. 104-116.

[8] L. Geissinger, Hopf algebras of symmetric functions and class functions, in Combinatoire et représentation du groupe symétrique (Strasbourg, 1976), Springer Lecture Notes in Math. 579, Springer-Verlag, New York, 1977, pp. 168-181.

[9] I. M. Gessel, Multipartite P-partitions and inner products of skew Schur functions, in Combinatorics and Algebra, Contemp. Math., Vol. 34, American Mathematical Society, Providence, 1984, pp. 289-301.

[10] Hoang Ngoc Minh, Des propriétés structurelles des polylogarithmes aux aspects algorithmiques des sommes harmoniques multiples, preprint. 
[11] F. Hirzebruch, Topological Methods in Algebraic Geometry, 3rd ed., Springer-Verlag, New York, 1966.

[12] M. E. Hoffman, Multiple harmonic series, Pacific J. Math. 152 (1992), 275-290.

[13] M. E. Hoffman, The algebra of multiple harmonic series, J. Algebra 194 (1997), $477-495$.

[14] M. E. Hoffman, Multiple zeta values and periods of mirrors, Proc. Amer. Math Soc. 130 (2002), 971-974.

[15] M. E. Hoffman, Algebraic aspects of multiple zeta values, in Zeta Functions, Topology, and Quantum Physics, Developments in Math., Vol. 14, Springer, New York, 2005, pp. 51-74.

[16] M. E. Hoffman, Quasi-symmetric functions and mod $p$ multiple harmonic sums, preprint arXiv:math.NT/0401319.

[17] A. Libgober, Chern classes and the periods of mirrors, Math. Res. Lett. 62 (1999), 193-206.

[18] D. E. Littlewood, The Theory of Group Characters and Matrix Representations of Groups, 2nd ed., Oxford University Press, London, 1950.

[19] R. Lu, The $\hat{\Gamma}$-genus and a regularization of an $S^{1}$-equivariant Euler class, preprint arXiv:0804.2714.

[20] I. G. MacDonald, Symmetric Functions and Hall Polynomials, 2nd ed., Oxford University Press, New York, 1995.

[21] C. Malvenuto and C. Reutenauer, Duality between quasi-symmetric functions and the Solomon descent algebra, J. Algebra 177 (1995), 967-982.

[22] K. McCadden, Analysis of the gamma function, USNA Honors Project, 2007.

[23] N. Nielsen, Die Gammafunktion, Chelsea, New York, 1965.

[24] R. P. Stanley, Enumerative Combinatorics, Vol. 2, Cambridge University Press, Cambridge, 1999.

[25] D. Zagier, Values of zeta function and their applications, in First European Congress of Mathematics, Vol. II (Paris, 1992), Birkhäuser, Basel, 1994, pp. 497-512. 


\section{Appendix: $\zeta, \zeta_{+}$and $\zeta_{-}$on Sym in monomial basis for weight $\leq 7$}

\begin{tabular}{|c|c|c|c|}
\hline & $\zeta$ & $\zeta_{+}$ & $\zeta_{-}$ \\
\hline$m_{(1)}$ & $\gamma$ & 0 & $\gamma$ \\
\hline$m_{(2)}$ & $\zeta(2)$ & $\zeta(2)$ & 0 \\
\hline$m_{\left(1^{2}\right)}$ & $\frac{1}{2} \gamma^{2}-\frac{1}{2} \zeta(2)$ & $-\frac{1}{2} \zeta(2)$ & $\frac{1}{2} \gamma^{2}$ \\
\hline$m_{(3)}$ & $\zeta(3)$ & 0 & $\zeta(3)$ \\
\hline$m_{(2,1)}$ & $\gamma \zeta(2)-\zeta(3)$ & 0 & $-\zeta(3)$ \\
\hline$m_{\left(1^{3}\right)}$ & $\frac{1}{6} \gamma^{3}-\frac{1}{2} \gamma \zeta(2)+\frac{1}{3} \zeta(3)$ & 0 & $\frac{1}{6} \gamma^{3}+\frac{1}{3} \zeta(3)$ \\
\hline$m_{(4)}$ & $\zeta(4)$ & $\zeta(4)$ & 0 \\
\hline$m_{(3,1)}$ & $\gamma \zeta(3)-\zeta(4)$ & $-\zeta(4)$ & $\gamma \zeta(3)$ \\
\hline$m_{\left(2^{2}\right)}$ & $\frac{3}{4} \zeta(4)$ & $\frac{3}{4} \zeta(4)$ & 0 \\
\hline$m_{\left(2,1^{2}\right)}$ & $-\gamma \zeta(3)-\frac{1}{4} \zeta(4)$ & $-\frac{1}{4} \zeta(4)$ & $-\gamma \zeta(3)$ \\
\hline$m_{\left(1^{4}\right)}$ & $\frac{1}{24} \gamma^{4}-\frac{1}{4} \gamma^{2} \zeta(2)+\frac{1}{3} \gamma \zeta(3)+\frac{1}{16} \zeta(4)$ & $\frac{1}{16} \zeta(4)$ & $\frac{1}{24} \gamma^{4}+\frac{1}{3} \gamma \zeta(3)$ \\
\hline$m_{(5)}$ & $\zeta(5)$ & 0 & $\zeta(5)$ \\
\hline$m_{(4,1)}$ & $\gamma \zeta(4)-\zeta(5)$ & 0 & $-\zeta(5)$ \\
\hline$m_{(3,2)}$ & $\zeta(2) \zeta(3)-\zeta(5)$ & 0 & $-\zeta(5)$ \\
\hline$m_{\left(3,1^{2}\right)}$ & $\frac{1}{2} \gamma^{2} \zeta(3)-\gamma \zeta(4)-\frac{1}{2} \zeta(2) \zeta(3)+\zeta(5)$ & 0 & $\frac{1}{2} \gamma^{2} \zeta(3)+\zeta(5)$ \\
\hline$m_{\left(2^{2}, 1\right)}$ & $\frac{3}{4} \gamma \zeta(4)-\zeta(2) \zeta(3)+\zeta(5)$ & 0 & $\zeta(5)$ \\
\hline$m_{\left(2,1^{3}\right)}$ & $\begin{array}{c}\frac{1}{6} \gamma^{3} \zeta(2)-\frac{1}{2} \gamma^{2} \zeta(3)-\frac{1}{4} \gamma \zeta(4) \\
+\frac{5}{2} \zeta(2) \zeta(3)-\end{array}$ & 0 & $-\frac{1}{2} \gamma^{2} \zeta(3)-\zeta(5)$ \\
\hline$m_{\left(1^{5}\right)}$ & $\begin{array}{c}\frac{1}{120} \gamma^{5}-\frac{1}{12} \gamma^{3} \zeta(2)+\frac{1}{6} \gamma^{2} \zeta(3)+\frac{1}{16} \gamma \zeta(4) \\
-\frac{1}{6} \zeta(2) \zeta(3)+\frac{1}{5} \zeta(5)\end{array}$ & 0 & $\frac{1}{120} \gamma^{5}+\frac{1}{6} \gamma^{2} \zeta(3)+\frac{1}{5} \zeta(5)$ \\
\hline
\end{tabular}




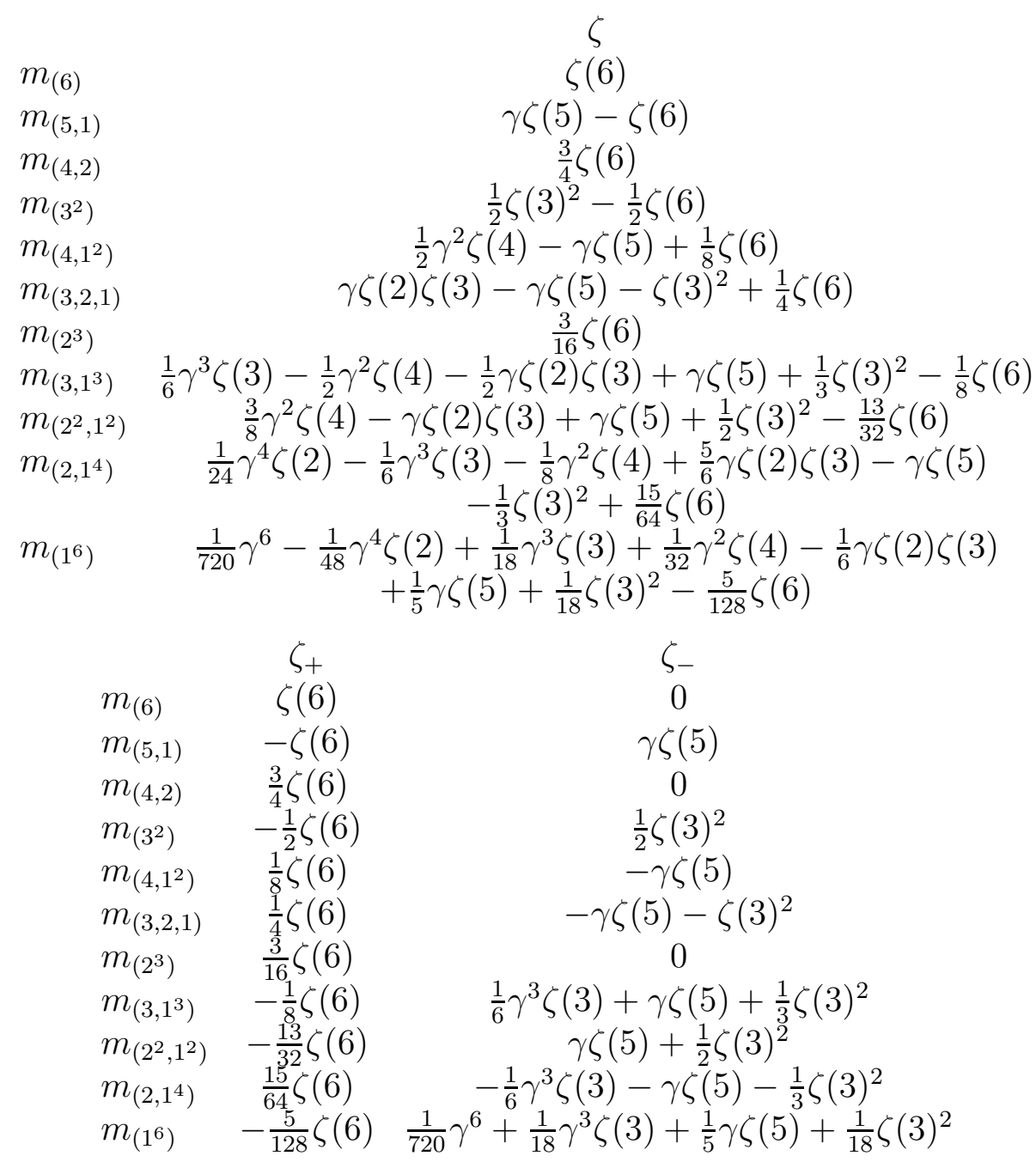




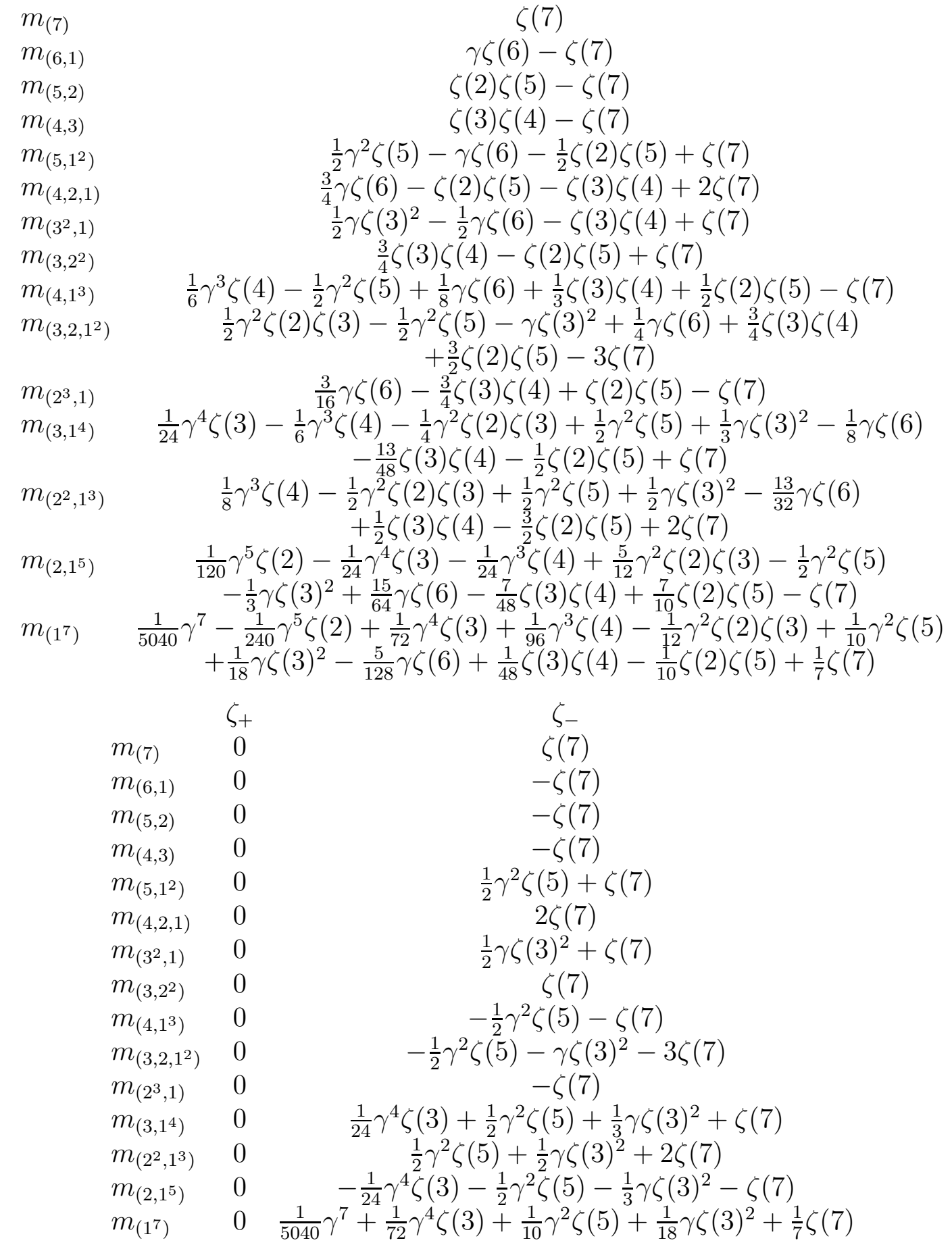

